\title{
Boull(é)e schreiben: \\ Thomas Bernhard, Peter Greenaway und die Architekturphysiognomik der Postmoderne
}

Wird auch in der Postmoderne noch shysiognomisch geschrieben ? Auf diese intrikate Frage findet sich in Architekturdiskursen der 1960er bis 1980er Jahre eine Antwort. Der Schriftsteller Thomas Bernhard, der Regisseur Peter Greenaway und die Architekten Hans Hollein, Walter Pichler und Aldo Rossi greifen auf die Architekturphysiognomik des 18. Jahrhunderts zurück. Ihre Architekturen sind im Sinne des französischen Revolutionsarchitekten Étienne-Louis Boullée eine sphysiognomische Schrift in doppelter Hinsicht: Sie sind das Resultat physiognomischer Beobachtungen und fordern als Baukörper eine physiognomische Lektüre ein.

In der Architektur, wo klar umrissene Theorie zu kultureller Praxis ausfranst, ist Physiognomik nicht primär als Wissenschaft konzipiert. Vielmehr soll sie konkrete ästhetische Aufgaben lösen. Hier hat sie sich schon vor ihrer Konjunktur bei Lavater und auch noch snach Auschwitz ${ }^{1}$ eher als Kulturtechnik denn als reflektierte Theorie ausgeprägt, weshalb sie den akademischen Niedergang physiognomischen Wissens überleben konnte. ${ }^{2}$ In der Moderne manifestierte sich das Interesse am `Gesicht der Zeit noch als Interesse am Gesicht der Architektur. ${ }^{3}$ Architekten wie Paul Schultze-

1 Auschwitz wird oftmals als Zäsur ausgemacht, mit welcher die Physiognomik endet. Vgl. z.B. Richard T. Gray: About face. German Physiognomic Thought from Lavater to Auschwitz, Detroit (Mich.) 2004. Dass eine solche Eingrenzung höchst problematisch ist, kritisiert z.B. Hans-Georg von Arburg in seiner Rezension zu Richard T. Gray: About Face. German Physiognomic Thought from Lavater to Auschwitz (2004), in: Germanistik. Internationales Referatenorgan mit bibliographischen Hinweisen 46/1-2 (2005), S. 143.

2 Daniela Bohde vermutet, dass die postmoderne Architektur »eine späte Erbin« der Architekturphysiognomik sein könnte. Daniela Bohde: Kunstgeschichte als physiognomische Wissenschaft. Kritik einer Denkfigur der 1920er bis 1940er Jahre, Berlin 2012, S. 4. Diese Anregung gilt es hier als These zu präzisieren und zu verifizieren.

3 So z.B. bei Walter Benjamin. Vgl. Detlev Schöttker: Raumerfahrung und Geschichtserkenntnis. Die "Architektur der Gesellschaft« aus Sicht der historisch-soziologischen Wahrnehmungstheorie: Giedion, Benjamin, Kracauer, in: Die Architektur der Gesellschaft. Theorien für die Architektursoziologie, hg. von Joachim Fischer und Heike Delitz, Bielefeld 2015, S. 137-162, hier S. 148. 
Naumburg und Adolf Behne sahen in der Physiognomik ein Werkzeug, die Gestalt einer Gesellschaft zu analysieren und zu gestalten. ${ }^{4}$ Postmoderne Adaptionen der Architekturphysiognomik rücken von solchen großen ideologischen Versprechen ab und konzentrieren sich auf den Ausdruck individueller Charakteristika. Die Architekturphysiognomik wandelt sich also von einer sozial-utopischen zu einer individuell-historistischen Kulturtechnik. Doch das Streben nach Individualität wird von einem anderen postmodernen Paradigma durchkreuzt: Die erhöhte Sensibilität für die historische Bedingtheit der eigenen Position ist gleichsam eine conditio sine qua non zur Abgrenzung von der Moderne.

Das Paradox, absolute individuelle Freiheit bei konsequenter historischer Selbstvergewisserung zu erlangen, erzeugt in der Postmoderne zwei architekturphysiognomische Strategien: die Appropriation historischer Ausdrucksformen (Einschreibung) und die Überbietung der historischen Formgebung (Überschreibung). Dies geschieht nicht zufällig unter Zuhilfenahme einer Schriftmetapher, die bereits in der frühen Architekturphysiognomik des 18. Jahrhunderts angelegt ist. Der Baukörper galt ihr als charakteristisches Zeichen, das der Architekten-Poet gleichsam mit Steinen schreibt. Der Bogen, der die Implikationen dieser physiognomischen Schriftmetapher im Folgenden nachzeichnet, beginnt darum bei den semiotischen Prämissen der französischen Revolutionsarchitektur, spannt sich über Werke Thomas Bernhards und Peter Greenaways und endet bei der postmodernen Architektur Hans Holleins, Walter Pichlers und Aldo Rossis.

Das Thema wird also zuerst über seine architekturtheoretische Grundlage des 18. Jahrhunderts und dann über deren Adaption in Literatur und Film 200 Jahre später erschlossen, um erst gegen Ende auf eigentliche Architekturentwürfe der Postmoderne zu sprechen zu kommen. Freilich hätte auch der umgekehrte Argumentationsweg beschritten werden können. Das gewählte Vorgehen ist jedoch einer Logik verpflichtet, welche vom expliziten zum impliziten Diskurs voranschreitet: Ist Physiognomik in der Theorie der Revolutionsarchitektur noch ein offensichtliches, wenn auch begrifflich abwesendes Hilfsmittel, so hat sich die postmoderne Architektur scheinbar längst von ihren wirkungsästhetischen Versprechen verabschiedet. ${ }^{5}$ Das

4 Vgl. Hans-Georg von Arburg: "Sage mir, wie du baust, und ich sage dir, wer du bist!« Physiognomik in der Architekturpublizistik der Weimarer Republik, in: Im Lichte Lavaters. Lektüren zum 200. Todestag, hg. von Ulrich Stadler und Karl Pestalozzi, Zürich 2003, S. 165-200.

5 Detlev Schöttker zufolge hat sich die postmoderne Architektur gar insgesamt von der Wirkungsästhetik abgewandt. Vgl. Detlev Schöttker: Architektur als Literatur. Zur Geschichte 
problematische Erbe der Physiognomik lebt dort nichtsdestotrotz subkutan fort. Bernhards und Greenaways Werke machen auf dieses Fortleben aufmerksam und nehmen damit eine vermittelnde Stellung im Aufbau dieses Aufsatzes ein: Im Medium des Romans und des Filmes werden die physiognomischen Prämissen einer ssprechenden< bzw. sgeschriebenen Architektur< in der Postmoderne zur Sprache gebracht.

\section{Die französische Architekturphysiognomik des 18. Jahrhunderts}

Physiognomisches Schreiben ist in seinem metaphorischen Sinne nicht auf das geschriebene Wort beschränkt; auch Film- ${ }^{6}$ Kunst $^{7}$ und Bautheorien suchten ihren Gegenstand als >Aufschreibesystem zu rechtfertigen, das ebenso physiognomischen Lektüren wie der physiognomisch motivierten Kunstproduktion offensteht. Insbesondere die architecture parlante von Étienne-Louis Boullée, einem französischen Architekten des späten 18. Jahrhunderts, versteht sich als Symbolschrift, die mit geometrischen Formen Charakteristisches festhält und dieses in der »impression" des Betrachters wieder entfaltet. ${ }^{8}$ Sie kombiniert ein abstraktes Formvokabular mit einer sensualistischen, d.h. im Sinne des empirischen Sensualismus das menschliche Empfindungsvermögen priorisierenden Wirkungsästhetik. ${ }^{9}$ Boullées Lehrer Jacques-François Blondel hat noch vor der Konjunktur Lavaters eine Lehre der caractères entwickelt, ${ }^{10}$ in der Gebäudeformen einen mensch-

und Theorie eines ästhetischen Dispositivs, in: Transmedialität: Zur Ästhetik paraliterarischer Verfahren, hg. von Urs Meyer und Roberto Simanowski, Göttingen 2006, S. 131151, hier S. 136f. Dass auch die postmoderne Architekturtheorie problematische wirkungsästhetische Annahmen aufweist, wird im fünften Abschnitt dieses Aufsatzes gezeigt.

6 Béla Balázs' physiognomisch grundierte Filmtheorie fasst die Arbeit des Monteurs als »Dichtung«, die der gefilmten »Wirklichkeit« mithilfe einer zweckmäßigen Anordnung von »Pantomime[n]«»Bedeutung« verleiht. Béla Balázs: Der Geist des Films, Halle 1930, S. 94.

7 Lottlisa Behling umschreibt die Ornamentik eines Kunstwerks gleichsam als Schrift, die es kunstphysiognomisch zu entziffern gilt. Vgl. Daniela Bohde: Kunstgeschichte als physiognomische Wissenschaft, S. 188.

8 Étienne-Louis Boullée: Architecture: Essai sur l'art, hg. von Helen Rosenau, London 1953, S. 41: "J'appelle caractère l'effet qui résulte de cet objet, et cause en nous une impression quelconque."

9 Zur Wichtigkeit des Sensualismus für verschiedene Lehren des architektonischen caractère vgl. Werner Szambien: Symétrie. Goût. Caractère. Théorie et terminologie de l'architecture à l'âge classique 1550-1800, Paris 1986, S. 198; ferner dazu auch Daniela Bohde: Kunstgeschichte als physiognomische Wissenschaft, S. 4.

10 So betont Blondel etwa in seinem Encyclopédie-Artikel zur "Façade«, dass der Architekt seinem Gebäude nicht jeden beliebigen »charactere [sic]« aufprägen dürfe. Jacques-François 
lichen Ausdruck imitieren und die Repräsentation eines Menschentypus, eines Bauherren oder Architekten gleichsam in Stein schreiben. ${ }^{11}$ Notabene verweist die Etymologie von caractère selbst auf das Schreiben zurück, besaß der Ausdruck doch bis ins 17. Jahrhundert noch die alleinige Bedeutung des Prägestempels und Schriftzeichens (und trägt diese Bedeutung im Englischen und Französischen weiterhin mit sich). Erst die Kunstanalyse des siebzehnten Jahrhunderts überträgt den Begriff auf die individuelle Handschrift des Malers und von dort wiederum auf die Individualität des Künstlers selbst. ${ }^{12}$ Der caractère-Begriff, den Boullée von seinem Lehrer übernimmt, vereint noch immer beide Bedeutungsdimensionen von Schrift-Form und individuellem Ausdruck. Es eröffnet damit ein bis heute wirkungsmächtiges architektursemiotisches Problem, dass Architektur sowohl über eine einfach lesbare Typologie als auch eine emotive Sprache verfügen soll, die ihrer intendierten Bedeutung und Wirkung angemessen ist. ${ }^{13}$ Die Semiotik des caractère sucht in diesem Sinne die räußere architektonische Form, also die Gebäudeform, als einen reproduzierbaren Signifikanten mit einem sinneren Signifikat, d.h. mit einem scharakteristischen Wesen, zu vereinen. Es kann sich bei diesem Signifikat um das Wesen einer Idee, eines Bewohners oder, wie im Falle von Boullées Newton-Denkmal (Abb. 1), auch um eine Einheit von Person und Ideal handeln.

Blondel: Art. "Façade«, in: Encyclopédie, ou dictionnaire raisonnée des sciences, des arts et des métiers, par une sociéte gens de lettres, hg. von Denis Diderot und Jean le Rond d'Alembert, Bd. 6, Paris 1751, S. 355. Vgl. hierzu auch Hans-Georg von Arburg: "Sage mir, wie du baust, und ich sage dir, wer du bist!«, S. 167.

Auf die Spitze treibt dieses physiognomische Bau-Denken eine Generation nach Boullée der Architekt und Zeichner Jean-Jacques Lequeu. Vgl. Anthony Vidler: The Writing of the Walls. Architectural theory in the late enlightenment, Princeton New Jersey 1987, S. 118-124. Zu Lequeu s.u., Anm. 24. Ähnlich argumentiert im selben Zeitraum eine anonyme Architekturschrift in Deutschland: Untersuchungen über den Charakter der Gebäude; über die Verbindung der Baukunst mit den schönen Künsten, und über die Wirkungen, welche durch diese hervorgebracht werden sollten, Leipzig 1788, FaksimileNeudruck, hg. von Hanno-Walter Kruft, Nördlingen 1986.

12 Vgl. Daniela Bohde: Kunstgeschichte als physiognomische Wissenschaft, S. 47-58. Ähnlich auch Carlo Ginzburg: Spurensicherung. Der Jäger entziffert die Fährte, Sherlock Holmes nimmt die Lupe, Freud liest Morelli - die Wissenschaft auf der Suche nach sich selbst, in: ders.: Spurensicherungen. Über verborgene Geschichte, Kunst und soziales Gedächtnis, Berlin 1983, S. 61-96, hier S. 78f.

13 Vgl. dazu insbes. Charles Jencks: The language of post-modern architecture, London 1977. Jencks betrachtet die postmoderne Architektur als persönliches Ausdrucksproblem, das es zu meistern gelte: "We may expect to see the next generation of architects using the new hybrid language with confindence» (ebd., S. 62). 


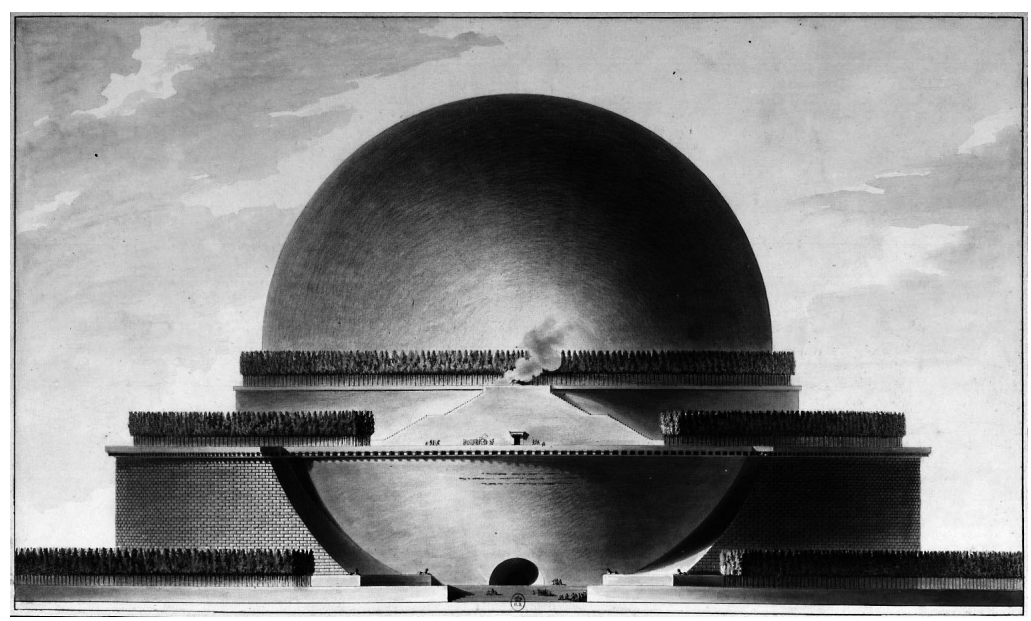

Abb. 1: Étienne-Louis Boullée, Cénotaphe à Newton (1795), Bibliothèque nationale, Paris

Die Sphäre des Leergrabes drückt sowohl den vollkommenen Charakter Newtons, seine wissenschaftliche Arbeit als auch das darauf beruhende, aufklärerisch-physikalische Weltbild aus und soll dieses dem Betrachter sensuell fassbar machen. ${ }^{14}$ Boullées Entwürfe blieben jedoch megalomanische Phantasien und gingen wie diejenigen seines Zeitgenossen und Mitstreiters Claude-Nicolas Ledoux alsbald vergessen.

Erst Emil Kaufmann popularisiert die vermeintliche Revolutionsarchitektur (die freilich schon vor der Französischen Revolution zu entstehen begann) 1933 in seinem Werk Von Ledoux bis Corbusier. ${ }^{15}$ Er stellt eine direkte Kontinuität zwischen dieser französischen Architektur des 18. und der modernen Architektur des 20. Jahrhunderts her. Die Architekturen Boullées beschäftigen Historiker, Architekten und Künstler seither nicht nur aufgrund ihrer die Moderne präfigurierenden Formen, sondern auch aufgrund des ihnen inhärenten Versprechens, reiner und sublimer Ausdruck höherer Ideen zu

14 Vgl. Étienne-Louis Boullée: Architecture: Essai sur l'art, S. 83-85. Dazu auch Adolf Max Vogt: Einführung, in: Étienne-Louis Boullée: Architektur. Abhandlung über die Kunst, hg. von Beat Wyss, Zürich/München 1987, S. 7-41, hier S. 29 f.

15 Emil Kaufmann: Von Ledoux bis Le Corbusier. Ursprung und Entwicklung der Autonomen Architektur, Wien 1933. 
sein. Neben Vertretern einer liberalen Moderne fand sie darum auch unter den Architekten des Faschismus große Bewunderer. ${ }^{16}$

Die produktive Auseinandersetzung mit Boullée hat sich in der Spät- und Nachmoderne fortgesetzt und so ein physiognomisches Denkmodell in die Postmoderne weitergetragen, während Physiognomik als Theoriekomplex nach 1945 beinahe vollständig diskreditiert bleibt. Architektur und Schrift, physiognomische Beobachtungs-, Bau- und Schreibtechnik werden in der postmodernen Boullée-Rezeption neu zueinander in Bezug gesetzt. Anhand zweier Beispiele soll im Folgenden der Frage nachgegangen werden, wie Boullées Bauen von caractère nun selbst um- oder überschrieben wird. Thomas Bernhards Roman Korrektur (1975) und Peter Greenaways Film The Belly of an Architect (1986) konfrontieren Boullées Werk mit eigenen physiognomischen Schreibprojekten. Die beiden Werke verhandeln gleichsam das Problempotential, welches das Konzept des caractère in postmodernen Tendenzen der Architektur entwickelt.

\section{Roithamer: Triumph der steinernen Schrift}

Das Gebäude, das der zurückgezogene, eigenbrötlerische Naturwissenschaftler Roithamer im Roman Korrektur baut, soll gänzlich seiner geliebten Schwester dienen. Entgegen dem Willen seiner beiden Brüder und der Schwester selbst entwirft Roithamer ihr das "vollkommen entsprechende[ ] "17 Wohnhaus, zugleich entfernt er die Geschwister vom verhassten Familiensitz Altensam. Diesen Eingriff überlebt die Schwester nicht. Kurz nachdem sihr Wohnkegel fertiggestellt ist, stirbt sie an einer nicht weiter beschriebenen Krankheit. Laut Roithamer habe gerade das »allerhöchste Glück (Ko 106) über den Kegel ihren Tod verursacht. In größter Einsamkeit, aber vehement behauptend, sein Lebensprojekt sei vollendet, nimmt sich der Architekt das Leben.

Ob Roithamers zweifelhafte Behauptungen zutreffen, kann der Leser von Korrektur schwer beurteilen. Die Geschehnisse werden unzuverlässig von einem namenlosen Freund berichtet, der lückenhaft aus Roithamers schriftli-

16 Vgl. Adolf Max Vogt: Revolutions-Architektur und Nazi-Klassizismus, in: Argo. Festschrift für Kurt Badt zu seinem 80. Geburtstag am 3. März 1970, hg. von Martin Gosebruch und Lorenz Dittmann, Köln 1970, S. 354-363.

17 Thomas Bernhard: Korrektur. Roman (1975), Werke, hg. von Martin Huber und Wendelin Schmidt-Dengler, Bd. 4, Frankfurt a.M. 2005, S. 99. Im Folgenden zitiert als Ko. 
chem Nachlass zitiert. Nach dem Tod der Schwester hat Roithamer nämlich versucht, Rechenschaft über seine Kindheit, seine Schwester und seinen Kegelbau in einer 800-seitigen Schrift abzulegen.

Trotz inkonsistenten Angaben zur Entstehung und zum Aussehen des Baus lässt sich rekonstruieren, welche historischen Vorbilder Roithamer rezipiert hat und was die theoretischen Grundlagen seines Bauens waren. In einer Suada über das Unwissen anderer Architekten behauptet Roithamer: WWenn wir handeln, kennen wir den Ursprung unseres Handelns, wenn wir denken, den Ursprung unseres Denkens. Boulle [sic], Hamilton, Vignon, Konzeptionswechsel etcetera, so Roithamer, wir reden vergeblich.«(Ko 186) Die Aufzählung der Architekten, in der »Boullée« an erster Stelle zum vielsagenden "Boulle« verstümmelt ist, verweist wie der Begriff »Konzeptionswechsel« auf Adolf Max Vogts Untersuchung Russische und Französische Revolutions-Architektur 19171789 (1974). ${ }^{18}$ Auf diese Untersuchung greift Roithamer auch an anderer Stelle zurück:

Und ich wußte, daß noch niemals vorher von einem Menschen, nicht einmal von einem französischen, nicht einmal von einem russischen, ein Kegel gebaut worden ist, mein Kegel wird der erste gebaute Kegel für Wohnzwecke sein, sagte ich mir und ich beschloß, den Kegel zu bauen. (Ko 192)

Mit der Abgrenzung gegenüber der Architekturgeschichte verstärkt er gleichzeitig das Band formaler Kontinuität und grenzt die Vorbilder seines Projektes ein. Pläne für einen kegelförmigen Bau liegen nämlich nur von Boullée und dem russischen Künstler Wladimir Tatlin vor. Dessen filigraner Gerüstturm Monument fir die dritte Internationale hat jedoch wenig Gemeinsamkeiten mit Roithamers schwerem, beinahe fensterlosem »Stein- und Ziegelbau (Ko 190). Étienne-Louis Boullées ungebauter Cénotaphe conique (Abb. 2) erfüllt weitgehend diese Eigenschaften.

18 Adolf Max Vogt: Russische und französische Revolutions-Architektur 1971 1789. Zur Einwirkung des Marxismus und des Newtonismus auf die Bauweise, Köln 1974. Josef König hat erstmals auf die Bedeutung der Revolutionsarchitektur in Korrektur hingewiesen, diese aber noch als rein politischen Verweis Roithamers gelesen, vgl. Josef König: "Nichts als ein Totenmaskenball«. Studien zum Verständnis der ästhetischen Intentionen im Werk Thomas Bernhards, Frankfurt a.M./New York 1983, S. 131f. Gernot Weiß hat nachgewiesen, dass ein Großteil der Architektennamen in Korrektur aus dem architekturtheoretischen Werk Max Vogts über die Revolutionsarchitektur stammt, vgl. Gernot Weiß: Auslöschung der Philosophie. Philosophiekritik bei Thomas Bernhard, Würzburg 1993, S. 81f. Vogts Buch liegt tatsächlich in der Nachlass-Bibliothek Thomas Bernhards im Thomas-Bernhard-Archiv in Wien, Forschungseinrichtung der Thomas Bernhard Privatstiftung, vor. 


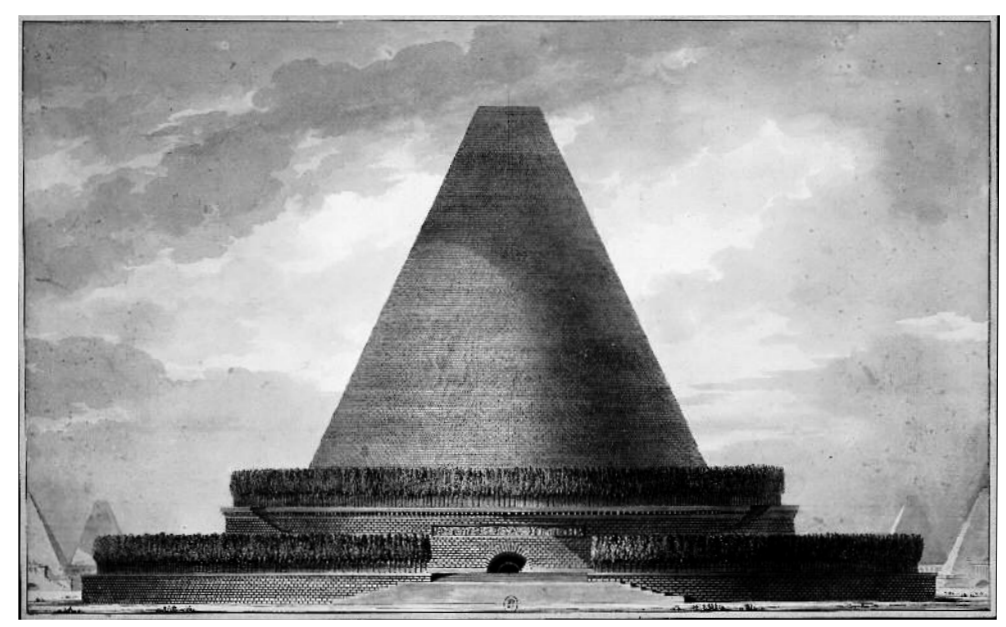

Abb. 2: Étienne-Louis Boullée, Cénotaphe conique (1782), Bibliothèque nationale, Paris

Wie schon mehrfach von der Forschung bemerkt, ${ }^{19}$ bildet der Cénotaphe als leeres Grabmonument Roithamers Gebäude auf tragische Weise vor. Der Kegel erinnert letztlich als leerstehende Ruine an den Tod seiner Schwester und gleicht so entgegen Roithamers anfänglicher Intention nicht nur der Form, sondern auch der Funktion von Boullées Kenotaphen. Bisher weitgehend unbeachtet blieben die architekturphysiognomischen Grundprämissen, welche Roithamer mit Boullée teilt. Der Kegel ist im Sinne von Boullées architecture parlante ein mit Stein geschriebener caractère: "Die Annahme, daß die Konzeption des Kegels genau dem Bedürfnis meiner Schwester, genau ihrem Charakter entspricht, ihrer Natur. [...] [D]er Kegel ist nicht, was sie zu dem jetzigen Zeitpunkt ist, er ist alles mit ihr Zusammenhängende." (Ko 292) Anders als bei Boullées Newton-Kenotaph, dessen runde Form auf die Ideenwelt des großen Physikers verweist, erfährt der Leser weiter nichts über die behauptete, auf den Charakter der Schwester abzielende `Entsprechung` des Kegels, außer dass ihr eine sehr genaue physiognomische Studie vorangegangen sei:

19 Vgl. z.B. Margarete Kohlenbach: Das Ende der Vollkommenheit. Zum Verständnis von Thomas Bernhards »Korrektur«, Tübingen 1986, S. 103f.; vgl. ferner auch Birgit Nienhaus: Architekturen und andere Räume. Raumdarstellung in der Prosa Thomas Bernhards, Marburg 2010, S. 121f., und Rike Felka: Das räumliche Gedächtnis. Untersuchungen zu Bernhard, Bachmann, Antonioni, Doderer, Stifter, Duras, Kafka, Berlin 2010, S. 1. 
Das Innere des Kegels wie das Wesensinnere meiner Schwester, das Äußere des Kegels wie ihr äußeres Wesen und zusammen ihr ganzes Wesen als Charakter des Kegels, aber Inneres und Äußeres des Kegels sind genausowenig voneinander trennbar, wie Inneres und Äußeres meiner Schwester, aber die unausgesetzte Beobachtung der Schwester und unausgesetzte Beobachtung der Konstruktion des Kegels haben zu dem Ergebnis geführt, das jetzt in der Mitte des Kobernaußerwaldes steht. (Ko 190)

Roithamers physiognomisches Projekt bleibt rätselhaft; warum gerade die Kegelform der Schwester entspreche, lässt sich weder anhand des Baus selbst noch mithilfe der ihm gewidmeten Schrift entschlüsseln. Des Letzteren ist sich Roithamer bewusst. Während seine Architektur fähig gewesen sei, den Charakter der Schwester zu fassen, sei ihm dies in der Schrift, die sich an ein größeres Publikum gerichtet habe, misslungen; die steinerne 'Schrift triumphiert traurig über das papierne Vermächtnis.

Dieses Missverhältnis ergibt sich notgedrungen aus Roithamers Präferenz der unmittelbaren Erkenntnis und ihrer intuitiven, genialen Übertragung in die Architektur. Solche Vorgänge können in der 800-seitigen Schrift nur schwer augenscheinlich werden, muss diese doch argumentativ rechtfertigen, was sich als charakteristische 'Entsprechung` nur in Stein ausdrücken lasse. Daraus erklärt sich auch Roithamers ostentative Ablehnung der eigenen architektonischen Vorbilder. Ihre angemessene Nennung würde den Kegel in eine nachvollziehbare Kontinuität stellen und damit der Behauptung widersprechen, Roithamer habe sein gesamtes genialisches Schaffen aus eigener Beobachtung geschöpft. Einzigartig und vollkommen kann sein Projekt nur dann sein, wenn dessen geschichtlicher Kontext verwischt wird: Der Name seines wichtigsten Vorgängers Boullée wird zu einem unbekannten und dennoch vielsagenden »Boulle« verkrüppelt. Denn »Boulle« spielt lautlich auf die monumentale architektonische 'Boule< Boullées an und lässt zugleich die gigantische Sphäre seines Newton-Kenotaphs zur lapidaren 'Kugek schrumpfen.

\section{Kracklite: Architektonische Vaterwünsche}

Genau diese Kugel steht neun Jahre nach Korrektur im Zentrum von Peter Greenaways Film The Belly of an Architect. Das nie gebaute Newton-Monument ist für den amerikanischen Architekten Stourley Kracklite eine der größten architektonischen Leistungen aller Zeiten. Sein Traum, eine Ausstellung über Étienne-Louis Boullée ausgerechnet in Rom zu kuratieren, begründet er denn auch mit dem Einfluss, welchen das Pantheon und der Petersdom auf den Franzosen gehabt haben. Die Kontinuität der Meisterwerke führt 
freilich auf direktem Wege zu Kracklite selbst. Zehn Jahre habe der 54-Jährige für die Planung der Ausstellung aufgewendet. Selbst baute er nur sechseinhalb Häuser, die der Zuschauer zudem nie zu Gesicht bekommt. Kracklites bisher größtes Bauwerk, ein Supermarkt für das Fleischwaren-Imperium seines Schwiegervaters in Chicago, bezeichnet er selbst als "a monument of carnivores ${ }^{20}{ }^{20}$ Es reihe sich in die »carnivorous architecture ${ }^{21}$ seiner Heimatstadt ein, die notabene eine Geburtsstätte der modernen amerikanischen Architektur ist. ${ }^{22}$ Auch ein anderer Bauauftrag an Kracklite stammt von seinem Schwiegervater; als Hochzeitsgeschenk sollte der Architekt sich und seiner Frau ein Haus bauen, das deren Vater finanziert. Entstanden seien, so Kracklites Frau, »two marble cubes and a brick sphere on stilts. Boullée would have loved it.«23 Die Beschreibung verweist ironischerweise mehr noch auf Boullées weniger bekannten, jüngeren Zeitgenossen Jean-Jacques Lequeu, der tatsächlich - bereits als ein Echo zu Boullées Entwürfen - eine "sphere on stilts« entworfen hat. ${ }^{24}$ In diesem Lichte ist Kracklites Haus gleich doppelt epigonal, die Nachahmung eines Nachfolgers.

Ebenfalls mithilfe seines Schwiegervaters stehen Kracklite nun eine Million Dollar und neun Monate zur Verfügung, um im Monumento a Vittorio Emanuele II die erste große Werkschau Boullées zu inszenieren. Der eklektischhistoristische Monumento kontrastiert die geometrische Klarheit von Boullées Architektur, teilt mit ihr aber eine schier maßlose Monumentalität. Der Bau beherbergt in seinem Zentrum den Altare della Patria. Die steingewordene Macht des Vaterlands ist ein Sinnbild für Kracklites eigene Vaterbeziehungen, seine doppelte Abhängigkeit vom Schwiegervater und vom Architektenvater Boullée. Letzterem schreibt Kracklite Postkarten, in denen er ihm seine privaten Probleme mitteilt. Seine Briefe an den toten Brieffreund enden

20 Peter Greenaway (Regie): The Belly of an Architect, England/Italien 1987, min. 00:06:50.

21 Ebd., min. 00:06:53.

22 Vgl. z.B. Charles Waldheim/Katharina Rüedi Rey: Chicago is History, in: Chicago Architecture: Histories, Revisions, Alternatives, Chicago 2005, S. XII-XXIII.

23 Peter Greenaway (Regie): The Belly of an Architect, min. 00:08:50.

24 Lequeu entwarf seinen temple consacré à l'Egalité 1793, acht Jahre nach Boullées NewtonMonument, vgl. Anthony Vidler: The Writing of the Walls, Abb. 88. Lequeu war zudem - wie Kracklite - ein weitgehend miss- und zum Teil verachteter Architekt seiner Zeit (vgl. ebd., S. 115). Seine Formsprache kann im Gegensatz zu derjenigen Kracklites aber als Ironisierung des architektonischen Symbolismus Boullées und Ledoux' verstanden werden (vgl. ebd., S. 118). Über beide verbreitete Lequeu üble Nachrede, zumal sie im Gegensatz zu ihm dem akademischen und aristokratischen Lager angehörten (vgl. ebd. S. 117). 
jeweils mit der Unterschrift "Stourley Kracklite (Architect) «, ${ }^{25}$ einer beinahe verzweifelten Behauptung angesichts seines geringen Erfolgs als Architekt. The Belly of an Architect lässt sich denn auch als Vater-Sohn-Drama verstehen. In seiner Unfähigkeit, sich vom historischen Übervater zu lösen, spiegelt sich Kracklites geistige Impotenz, selbst zum schöpferischen Vater zu werden. ${ }^{26}$ Biologischer Vater in spe wird er hingegen am Anfang des Filmes, als er über die Grenze Italiens fährt und im Nachtabteil mit seiner jungen Frau schläft. Peter Greenaways konzeptuelle Verschränkung von biologischer und geistiger Vaterschaft entwickelt im Folgenden massive Reibungen. In den neun Monaten der Schwangerschafts- und Konzeptualisierungszeit entgleiten Kracklite sowohl seine Ausstellung als auch seine Frau. Caspasian, ebenfalls Architekt und Sohn eines wichtigen Geldgebers, soll die Finanzierung des Projektes gewährleisten, veruntreut jedoch immer mehr Geld. Zugleich nimmt er sich Kracklites vernachlässigter Ehefrau an. Diese ist frustriert darüber, dass ihr Mann noch nicht einmal die Schwangerschaft bemerkt. In der exakten Mitte des Filmes erhält Kracklite Gewissheit über die Affäre, als er seine Frau beim Liebesspiel mit Caspasian ertappt. Unmittelbar zuvor beobachtet er, wie Caspasian mit einem Leuchtturm-Modell Boullées herumalbert, das er sich als Phallussymbol vor den Schritt hält (Abb. 3).

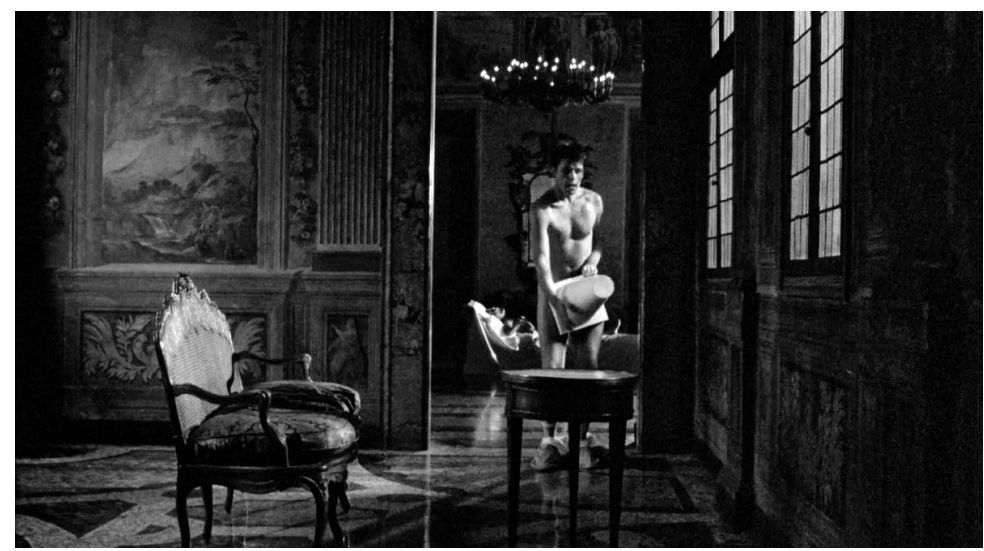

Abb. 3: Filmstill: Peter Greenaway, The Belly of an Architect (1987), DVD

25 Peter Greenaway (Regie): The Belly of an Architect, min. 00:29:58.

26 Diese Impotenz wird in der Sekundärliteratur immer wieder betont, vgl. z.B. Nancy Levinson: Tall Buildings, Tall Tales: On Architects in the Movies, in: Architecture and Film, hg. von Mark Lamster, Princeton 2013, S. 11-47, hier S. 34. 
Die Szene deutet an, dass Kracklite nicht nur seine Frau, sondern auch sseinen Boullée und damit seine Ausstellung an den Gegenspieler Caspasian verlieren wird, ist dieser doch eine überzeichnete jüngere und virilere Version des Vatersohnes.

Kracklite wird von schmerzhaften Magenkrämpfen geplagt. Er entwickelt eine Obsession für die Abbildung männlicher Bäuche, insbesondere desjenigen des römischen Kaisers Augustus, und für eine vermeintliche Darstellung Boullées. ${ }^{27}$ Mit einem Fotokopierer verhundertfacht er diese in mehrfacher Hinsicht vorbildlichen Abdomen, vergleicht sie mit ebenso zahlreichen Fotokopien seines eigenen Bauches und verortet auf ihnen die verspürten Schmerzen (Abb. 4).

Dem nackten männlichen Bauch steht der verhüllte Mutterbauch von Kracklites Frau gegenüber. Als sie ihm Schwangerschafts-Aktfotografien zeigen will, beginnt der Architekt ob deren vermeintlicher Obszönität zu toben. Die männlichen Bäuche der >Väter` Augustus und Boullée, die ebenfalls männliche Macht verkörpernden Kuppel-Gebäude Roms und Étienne-Louis Boullées Newton-Kenotaph werden zu Kracklites fetischistischen Sinnbildern seiner bedrohten, männlichen Schöpfungskraft. Architektur scheint ihm die männliche Unfähigkeit, ein Kind auszutragen, zu kompensieren. Kracklite liest die Bauch-Architekturen als idealisierten Ausdruck seines eigenen phy-

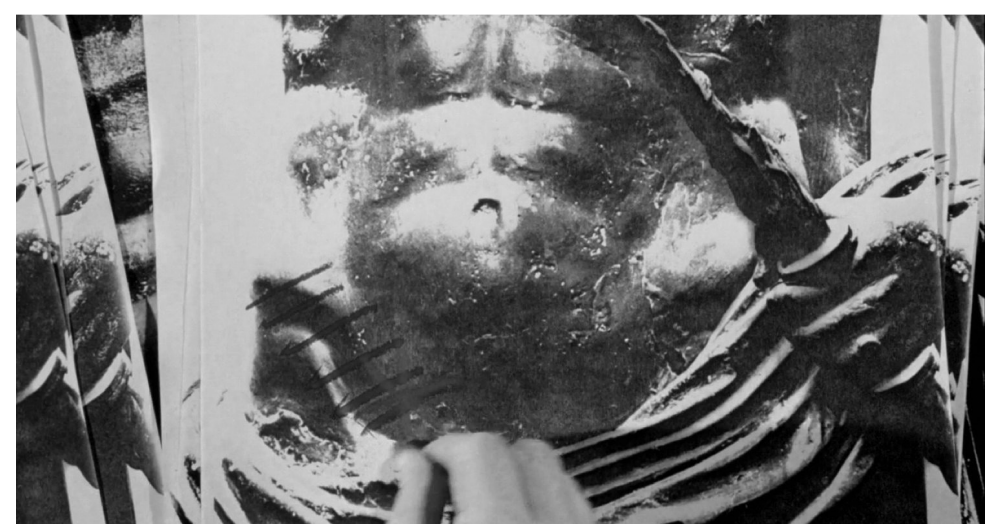

Abb. 4: Filmstill: Peter Greenaway,

The Belly of an Architect (1987), DVD

27 Bei der Abbildung, die ihm von Caspasian als Boullée-Porträt unterschoben wird, handelt es sich, wie Kracklite vermutet, wahrscheinlich um eine Fälschung mit den Zügen Piranesis. Piranesi ist denn auch eine Gegenfigur zu Boullée, auf die im Film immer wieder angespielt wird, vgl. Amy Lawrence: The Films of Peter Greenaway, Cambridge 1997, S. 130. 
sischen und psychischen Inneren, er unternimmt den Versuch einer physiognomischen Deutung ihres caractère.

Kurz vor Abschluss der Ausstellungsvorbereitungen erhält Kracklite Aufklärung über seine Magenschmerzen; der Bauchspeicheldrüsenkrebs ist so weit fortgeschritten, dass der Architekt den Frühling nicht überleben wird. Der selbsternannte Erbauer einer carnivorous architecture wird von innen aufgefressen. Angesichts seiner Krankheit und seiner geistigen Zerrüttung entzieht man ihm die Leitung der Ausstellung und überträgt sie Caspasian. Als unbemerkter Gast wohnt Kracklite der Ausstellungseröffnung bei, die am Geburtstag Boullées zu dessen Wiedergeburt beitragen soll. Kracklite stürzt sich auf dem Höhepunkt der Feierlichkeiten aus einem Fenster in den Tod. Just in diesem Moment erleidet seine Frau inmitten der Gäste eine Sturzgeburt und bringt vor dem geöffneten Modell des Newton-Kenotaphs Kracklites Kind zur Welt.

\section{Korrektur vs. The Belly of an Architect: Einschreiben und Überschreiben}

Wie schon Roithamers Selbstmord ist Kracklites Fall Triumph und Tragödie. Sein Lebenswerk ist vollbracht, er selbst ist in doppelter Hinsicht zum Vater geworden. Aber die Freude dieser Vaterschaft ist vergällt, das Lob für die Ausstellung wird sein Widersacher ernten, das Kind, so deutet es Kracklites Frau schon früher an, soll bei ihren Eltern, bei dem mächtigen Schwiegervater aufwachsen. Kracklite hat sich weder in eine kulturelle Kontinuität als Architekt und Ausstellungsmacher noch in eine biologische Kontinuität als Familienvater einschreiben können.

Bernhards und Greenaways Figuren bleibt damit nicht die Fertigstellung, aber der erwünschte Effekt ihrer Lebenswerke verwehrt. Ihre architektonischen caractères gehen fehl: Deren äußere Form bleibt ohne die intendierte Bedeutung, weil das äußerlich manifestierte 'Innen sich als Illusion erweist; die steinerne Schrift entwickelt nicht die angestrebte sensualistische Wirkung. Roithamers Glückskegel wird zum Kenotaph, sein Ausdruck teilt sich niemandem mehr mit. Stourley Kracklites Vorgehen, ein architektonisches Zeichen zu setzen, ist demjenigen Roithamers geradezu entgegengesetzt. Er sucht nicht die private Erlösung im Ausdruck seiner selbst und eines geliebten Menschen, sondern die öffentliche Selbstbehauptung als Architekt und Ausstellungsmacher. Die Paradoxie, Eigenes zu schaffen ohne eigene kreative Ideen, kann nur überwunden werden, wenn Kracklite mit Boullée identisch wird, sich ihm in einer einseitigen Brieffreundschaft immer mehr annähert. Wenn Kracklite etwa zu wissen behauptet, Boullée hätte die Farben Grün 
und Blau verabscheut, fußt dies auf dem Fantasma, wie Boullée zu sein und damit Boullées Werk noch einmal erschaffen zu dürfen.

Im Zuge dieser Re-Produktion verschiebt sich die ebenso neu gesetzte Bedeutung von Boullées zentralem Bau, seinem ikonischen Kugel-Monument. Ist dieses anfänglich Ausdruck von Kracklites schöpferischer Vaterwerdung, seinem Substitut für den Mutterbauch und damit seinem Aufstieg in die Reihe der großen Kuppel-Erbauer, so wird es wie Roithamers Kegel zum Symbol des Todes, zum Abbild von Kracklites ’krebsschwangerem Bauch. Im Gegensatz aber zur plötzlichen Neudeutung des Kegels, die der Tod von Roithamers Schwester ausgelöst hat, geschieht der Umschwung in Kracklites Bedeutungszuweisung schrittweise. Zeitgleich wird das Newton-Monument von anderen Instanzen mit neuen und widersprüchlichen Bedeutungen aufgeladen. Zum Ende steht der Modell-Kenotaph nicht alleine für Kracklites Tod, sondern auch für die Niederkunft seiner Frau und die triumphale Aneignung Boullées durch Caspasian. Das halb offene, aus seinem Innern leuchtende Modell erzeugt schließlich einen offensichtlichen Bedeutungsüberschuss, der Kracklites Deutungshoheit zuwiderläuft. Dies steht in fundamentalem Kontrast zum unterdeterminierten Kegel Roithamers.

Verbunden mit dem unterschiedlichen Ausmaß, in welchem die beiden Architekten über die Determinierung sihrer ^Architektur verfügen, ist ihre Einbindung in architekturgeschichtliche Kontinuitäten: Roithamers manipulativer Schriftgebrauch, der dem Leser die Bedeutung seines Kegels vorenthält, geht einher mit seiner Abwehr gegen die Architekturgeschichte. Er schreibt Boullées Namen zu 'Bouller um und überschreibt damit die geschichtliche Quelle. Demgegenüber ist Greenaways Architekt Kracklite davon besessen, Boullée seine Referenz zu erweisen, seine Deutungsohnmacht entspricht der Macht, welche die Architekturgeschichte auf ihn ausübt. Der Monumento a Vittorio Emanuele II, auf dessen >Vaterlandsaltar Kracklite sein Leben opfert, wird von den Römern abschätzig als sSchreibmaschine bezeichnet. Das Gebäude wird auch für Kracklite gleichsam zur Schreibmaschine, mit der er sich in die Geschichte einschreiben will. Dies macht Greenaways symbolische Filmsprache explizit; im Atelier Kracklite steht ein Modell des Monumento unmittelbar neben einer Schreibmaschine. Das Medium Film verweist hier auf die mediale Vermittlungsleistung von Architektur.

Eine vertiefte Reflexion über Medialität lässt sich sowohl in Korrektur als auch in The Belly of an Architect konstatieren. Von Kracklites Einschreibung und Roithamers Überschreibung zeugen jeweils ihre Schriften, welche die architektonischen Unterfangen komplettieren und reflektieren. Kracklites Briefe und Roithamers Rechtfertigungen verfehlen aber wie die Architekturen 
ihre Wirkung. Sie vermitteln dem Leser bzw. dem Zuschauer hingegen einen Eindruck, was es heißen kann, Boullée oder eben nur ’Boulle zu schreiben: Die beiden Architekten binden sich auf tragische Weise an die architektursemiotischen Prämissen Étienne-Louis Boullées. Anders als das moderne Paradigma reiner Funktionalität (ein Ansatz, der freilich seine eigenen Tücken birgt), kann die Kopplung der geometrischen Formensprache an eine physiognomisch-sensualistische Wirkungsästhetik keine nachvollziehbare Rationalität und keine wissenschaftliche Überprüfbarkeit für sich geltend machen. Ob und wie die Zeichensprache des caractère ihr Ziel erfüllen kann, ist von unüberschaubaren und unkontrollierbaren Begleitumständen abhängig, die größtenteils subjektiver Natur sind. Eine caractère-Architektur will nicht nur funktional genutzt, sie will sgelesen und sinnlich rezipiert werden, um dadurch in ihrem Rezipienten kognitive und emotionale Prozesse auszulösen. Dies scheitert, wo die architecture parlante aufgrund fehlender 'Sprachkenntnisser nicht verstanden wird, sich ihre Botschaft durch manipulierte oder zufällige Ereignisse ändert oder wo ihr schlicht die vorgesehenen Rezipienten fehlen.

\section{Hans Hollein, Walter Pichler und Aldo Rossi}

Die produktive postmoderne Boullée-Rezeption durch die Architektur ist vor ähnliche Probleme wie diejenigen Kracklites und Roithamers gestellt. Denn mit der Appropriation der französischen Revolutionsarchitektur und ihrem Versprechen, in geometrischen Formen Charakteristisches auszudrücken, werden auch die Schwierigkeiten physiognomischer Ausdrucksleistung übernommen. Ohne dass eine Reflexion der Physiognomik geschieht, wie Korrektur und The Belly of an Architect sie leisten, wird damit ein Diskurs fortgesetzt, der als längst beendet gilt.

Roithamer behauptet, seine Architektur verfüge über die absolute Macht, zu vermitteln, was er als Architekt intendiert - sei dies das vollkommene Glück seiner Schwester oder schlicht »alles mit ihr Zusammenhängende« (Ko 292). Eine solch eigenständige Kraft einer »Absoluten Architektur «28 propagiert auch der Architekt Hans Hollein, der in den 1960er Jahren mit seinen provokanten Texten und ausgefallenen Entwürfen die Postmoderne Österreichs

28 Hans Hollein: [Architektur ist eine geistige Ordnung], in: Architektur, Work in Progress, Ausstellungskatalog Galerie St. Stephan, 8-12. Mai, Wien 1963, S. 6, zit. nach: The Austrian Phenomenon. Architektur Avantgarde Österreich 1956-1973, hg. von Dietmar Steiner, Wien 2009, S. 20. 
einläutet. Hollein verfolgt ein Gegenprogramm zu den modernen Paradigmen der Funktion, der Transparenz und der leichten Bauweise. Er verzichtet weitgehend darauf, an bestimmte Baustile anzuknüpfen. Stattdessen soll Architektur wie die Pop-Art unmittelbar zu ihren Betrachtern sprechen: „Ein Bauwerk", so Hollein, "soll zeigen, was es bedeutet«.${ }^{29}$ Holleins Monument für die Opfer des Holocausts von 1963 (Abb. 5) weist große Ähnlichkeit zu Boullées Grab der Spartaner auf (Abb. 6).

Der `Hoftheoretiker der jungen Wiener Postmoderne, Günther Feuerstein, zieht 1965 denn auch Parallelen zwischen Holleins Entwürfen und der französischen Revolutionsarchitektur: »Ledoux [...] zeichnet Häuser in Form von Kugeln, Pyramiden, Ringen. Der Symbolwert der Form bestimmt das Gebäude. Es kann für irgend etwas benützt werden. Oder auch gar nicht

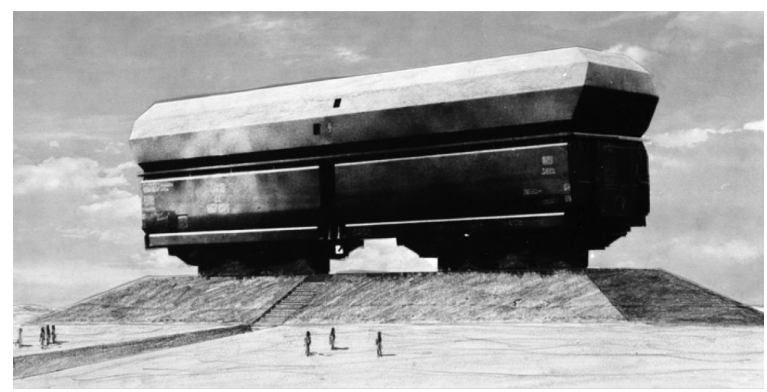

Abb. 5: Hans Hollein, Monument für die Opfer des Holocausts (1963) @ Nachlass Hollein

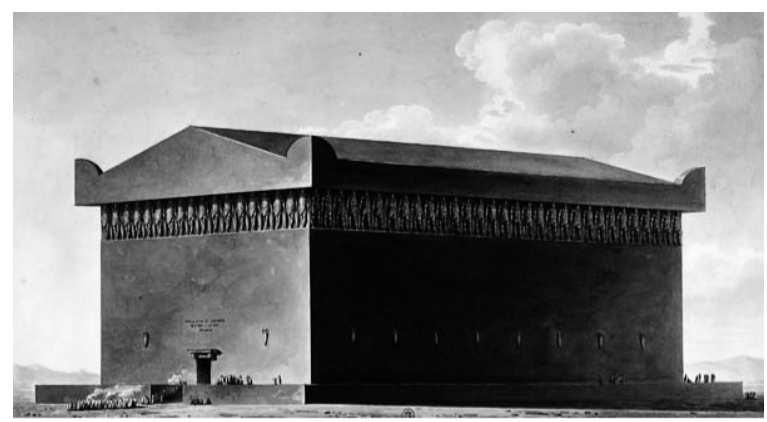

Abb. 6: Étienne-Louis Boullée, Tombeau des Spartiates (1782), Bibliothèque nationale, Paris

29 Ders.: Zurück zur Architektur [Ausschnitte aus einem Vortrag in der Galerie St. Stephan, Wien, 1962], in: Bau. Schrift für Architektur und Städtebau 24/2-3 (1969), S. 5-6, hier S. 5. 
benützt werden. Es bleibt aber Architektur. « ${ }^{30}$ Laut dem Architekten Joseph Esherick, einem weiteren Freund der Szene, will Hollein mit einer "Sprache" der »klaren und reinen Ausdrücke[ ] [...] ewige[n] Tatsachen" eine Form verleihen. ${ }^{31}$ Damit wiederholt er das Paradigma der architekturphysiognomischen caractères, die ein abstraktes Innen< in äußere Form übersetzen sollen. Im Gegensatz zu Boullée oder Architekturphysiognomen der Moderne wird bei Hollein jedoch kein kritisches politisches Konzept erkennbar, sein absoluter Anspruch auf Ausdruck kann später ebenso zum Bau eines Kerzengeschäfts oder einer Bijouterie dienen: Der plakative "Symbolwert" dient hier dem Verkauf. ${ }^{32}$ Im Falle von Holleins ungebautem Holocaust-Monument, einem überdimensionalen, geometrisch vereinfachten Eisenbahnwagon, stellt sich freilich die Frage, inwiefern der reine Ausdruck ^ dieser Architektursprache ihrem Gegenstand gerecht wird oder aber in Kitsch abdriftet.

Einen anderen Weg beschreitet Holleins Freund und architektonischer Mitstreiter Walter Pichler. Pichlers geometrische, meist halb unter die Erde versetzten Strukturen stellen ähnlich dem Kegel Roithamers keine explizite semantische Referenz her, suggerieren jedoch Analogien zwischen Architektur, menschlichem Körper und emotionalem Ausdruck, paradigmatisch etwa in Pichlers das Haus Oggau (Abb. 7).

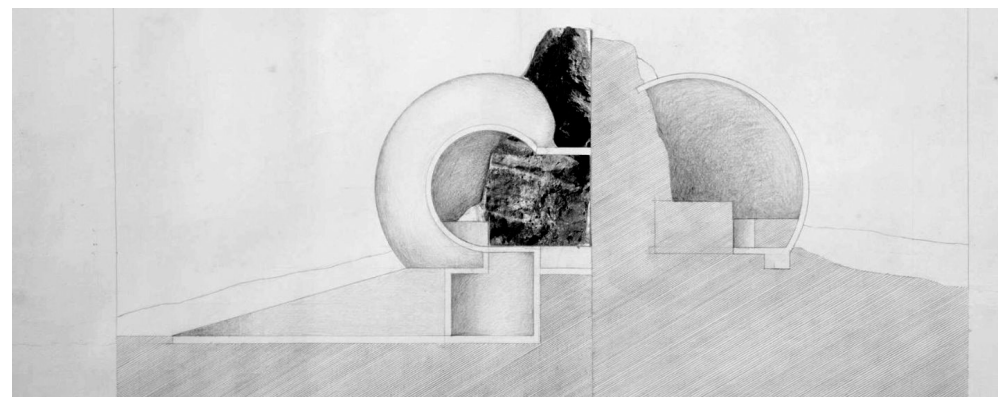

Abb. 7: Raimund Abraham/Walter Pichler, House, Oggau, Burgenland, Austria (1963). Graphite with collaged photographs on paper; 12 7/16 in. x $263 / 4$ in. $(31.59 \mathrm{~cm} \times 67.95 \mathrm{~cm})$; San Francisco Museum of Modern Art, Accessions Committee Fund purchase (C) Abraham / Pichler

30 Günther Feuerstein: Thesen zu einer Inzidenten Architektur (1958), in: Bau. Schrift für Architektur und Städtebau 24/2-3 (1969), S. 3-4, hier S. 3.

31 Joseph Esherick: [Einleitung], in: Architektur, S. 3-4, hier S. 4, zit. nach: The Austrian Phenomenon, S. 18.

32 Der Kerzenladen Retti von 1966 und das Juweliergeschäft Schullin 1 von 1974 sind nur die beiden berühmtesten Beispiele von Holleins Architekturen des (Selbst-)Verkaufs, vgl. http:// www.hollein.com/ger/Architektur/Nach-Typus/Shops-und-Interiors (22. Dezember 2015). 
Die Frage, wie ihre Formen im Detail zu lesen sind, bleibt unklar bzw. wird von Pichler bewusst verunklärt: "Architektur ist « laut dem Architekten die »Verkörperung der Macht und Sehnsüchte weniger Menschen«. ${ }^{33}$ Als enigmatische »Verkörperungen" geben Pichlers Entwürfe nur noch zu erkennen, dass hier etwas als physiognomischer Ausdruck zu lesen wäre, würde man zum Kreis der Wenigen gehören, an die dieser sich richtet. Dass Roithamers Kegel-Projekt die tödlichen Konsequenzen einer solchen elitären und hermetischen Konzeption von Architektur aufzeigt, muss kein Zufall sein: Walter Pichler und Thomas Bernhard haben sich gekannt, ihre Werke entstanden in nahen topografischen, historischen und sozialen Kontexten. ${ }^{34}$ Trotzdem gilt es, die Differenzen nicht außer Acht zu lassen: Bei der frühen Architektur Walter Pichlers und Hans Holleins handelt es sich wie bei vielen Plänen Boullées und Ledoux' um Entwürfe, die gar nie gebaut werden sollen. Damit entziehen sie sich dem möglichen Scheitern ihrer Zeichenhaftigkeit, das in Korrektur fiktional vollzogen wird.

Der postmodernen Tendenz einer rabsoluten Architektur steht eine Postmoderne gegenüber, die ihre historische Reflexion betont. Einer ihrer wichtigsten Vertreter ist Aldo Rossi, der schon zu Beginn seiner Laufbahn Boullées Essai sur l'art erstmals auf Italienisch übersetzt. ${ }^{35}$ Wie der fiktive Architekt Stourley Kracklite ist Aldo Rossi beflissen, Boullées Werk zurück in einen geschichtlichen Kontext einzubetten und damit auch sein eigenes Schaffen in diese Kontinuität zu stellen. Präsent wird dieser Wunsch im Wandgemälde La città analoga, das Rossi 1973 bei seinem Schüler Arduino Cantàfora in Auftrag gab. ${ }^{36}$ Im Fluchtpunkt des programmatischen Bildes befindet sich Boullées Leuchtturm-Entwurf, im zentralen Vordergrund Rossis Monumento ai Partigiani, ikonische Gebäude der Architekturgeschichte säumen die Straße, welche sie verbindet (Abb. 8).

33 Hans Hollein/Walter Pichler: [Architektur/Sie wird geboren], in: Architektur, S. 16.

34 Eine Kurzgeschichte Bernhards erschien gleichsam als Nachwort in einem ZeichnungenKatalog Pichlers, vgl. Thomas Bernhard: Ebene, in: Walter Pichler. 111 Zeichnungen. Mit einem Essay von Max Peintner und einem Prosatext von Thomas Bernhard, Salzburg 1973, S. 245-247. Die Bekanntschaft zwischen Pichler und Bernhard scheint jedoch eher oberflächlich gewesen zu sein, da sich bislang keine weiteren Dokumente hierzu fanden. Die Publikation der Kurzgeschichte war vermutlich ein Freundschaftsdienst gegenüber dem gemeinsamen Salzburger Residenz Verlag.

35 Étienne-Louis Boullée: Architettura saggio sull'arte, hg. und übersetzt von Aldo Rossi, Venedig 1967.

36 Vgl. Arduino Cantàfora: La città analoga (1973), in: Aldo Rossi/Gianni Contessi: Arduino Cantàfora, Mailand 1984, S. 145. 


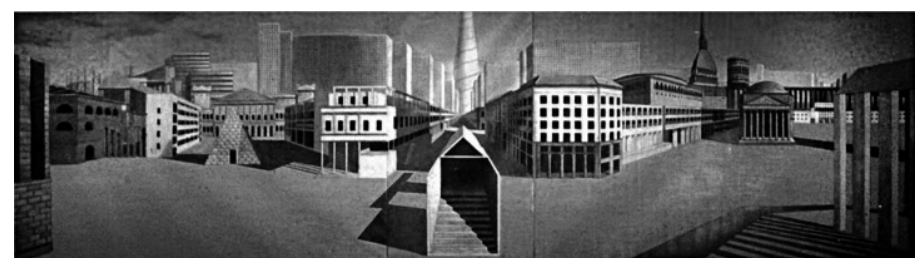

Abb. 8: Arduino Cantàfora, La città analoga (1973)

(C) Arduino Cantàforas

Anders als Kracklite markiert Rossi zwar sehr wohl Differenzen zu Boullée, dies relativiert aber nicht den Einfluss, welche die Revolutionsarchitektur auf das Formenrepertoire und Denken Rossis gehabt hat. Trotz betont empirischem Fundament behauptet auch er einen subjektiven und emotionalen architektonischen Ausdruck: Architektur sei »eine Art, die grundlegende Suche nach dem Glück zum Ausdruck zu bringen «. ${ }^{37}$ In diesem Sinne interpretiert Rossi seine eigenen Gebäude als »Formen, in denen sich das Leben und der Tod manifestier[en] «, 38 und erwähnt in seiner Autobiografie eine Vielzahl autobiografischer Erlebnisse, Lektüren und Bauten, die letztlich zur Formgebung beitrugen. ${ }^{39}$ La Nuova Piazza in Perugia (Abb. 9), eines seiner wichtigsten Bauten, spricht eine monumentale, aber historisch reflektierte geometrische Formensprache.

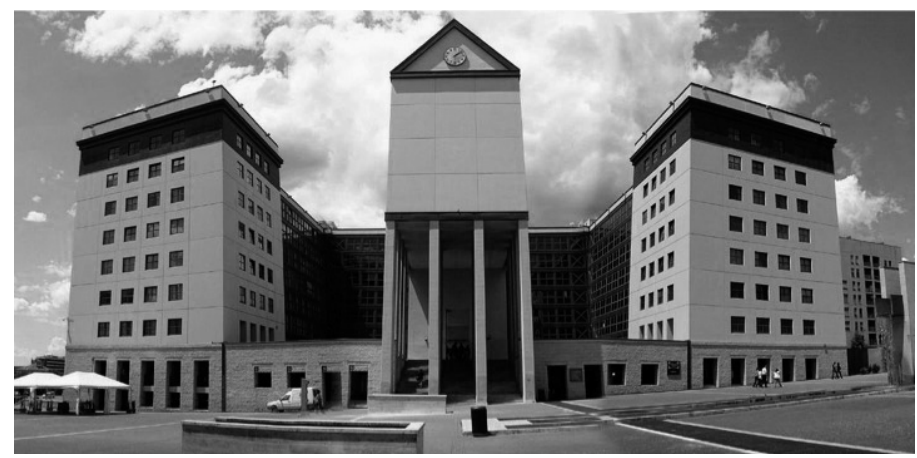

Abb. 9: Aldo Rossi, La Nuova Piazza Perugia (1984)

(C) Dave Morris

37 Aldo Rossi: Wissenschaftliche Selbstbiographie, übersetzt von Heinrich Helfenstein, Bern/ Berlin 1988, S. 12. Die Erstausgabe erschien 1981.

38 Ebd., S. 126.

39 Darunter auch Boullées Schaffen und Rossis persönliche Auseinandersetzung mit diesem, vgl. ebd., S. 82 und 137. 
Welcher persönliche Ausdruck sich hinter dieser verbirgt, bleibt freilich nicht rekonstruierbar. Ausgehend von Peter Greenaways Film, stellt sich auch bei dieser Architektur die Frage, ob sie sich mithilfe ihrer ostentativen historischen Referenz in die Architekturgeschichte einzureihen vermag oder von dieser nicht gleichsam überdeckt wird.

The Belly of an Architect und Korrektur reflektieren also nicht nur das Potential einer Architekturtheorie der caractères, sie weisen damit zugleich auf Probleme scharakteristischer Architektur ihrer Zeit hin. Was heißt dies für den Fortbestand der Physiognomik als Kulturtechnik der Architektur in der Postmoderne? Die Architekturphysiognomik der Revolutionsarchitekten verfolgt ein sensualistisch-aufklärerisches Programm, und die moderne Architekturphysiognomik strebt nach der Erfüllung sozialer Utopien. Danach findet ein Rückzug auf den innovativen, authentischen Ausdruck statt, der nicht länger einer klar umrissenen Ideologie entspricht und darum auch gänzlich dem einzelnen Individuum verpflichtet sein kann. Die neuen Herausforderungen einer postmodernen Architekturphysiognomik verschärfen Thomas Bernhard und Peter Greenaway in ihren je eigenen Medien zu Aporien. Denn hier werden architekturphysiognomische sSchreibprojekter vor ein schwerwiegendes Dilemma gestellt: Einerseits droht der architektonische Ausdruck unlesbar zu werden, wenn er historische Formensprachen radikal zu überschreiben sucht, andererseits begibt er sich in Gefahr, von der Architekturgeschichte selbst überschrieben zu werden, wenn er nur noch deren Sprache spricht. 